\title{
Towards a Gigahertz Microfluidic Impedance Cytometer
}

\author{
Niels Haandbæk, Sebastian C. Bürgel, Flavio Heer, and Andreas Hierlemann \\ ETH Zürich, Department of Biosystems Science and Engineering, Basle, Switzerland, \\ niels.haandbaek@bsse.ethz.ch
}

\begin{abstract}
:
We present a microfluidic impedance cytometer enabling dielectric characterization of single cells at frequencies up to $500 \mathrm{MHz}$, which represents a more than ten-fold frequency increase compared to other devices. The dielectric properties at lower frequencies reveal information about cell size and membrane capacitance. The increased frequency range of the presented cytometer potentially allows for characterization of subcellular components such as vacuoles or the cell nuclei. We demonstrate the capabilities of the cytometer by discriminating polystyrene beads from Chinese hamster ovary $(\mathrm{CHO})$ cells, based on the difference in dielectric properties at frequencies up to $500 \mathrm{MHz}$. The low frequency data show similar responses of cells and beads, which indicate that they are of comparable size. The high frequency results show a smaller response of the cells, due to the higher conductivity of cytoplasm and nucleus in comparison to the non-conducting beads.
\end{abstract}

Key words: Microfluidics, single-cell, flow-through, impedance spectroscopy, high frequency

\section{Introduction}

Impedance spectroscopy is a non-invasive technique for measuring the dielectric properties of a material of interest. It has found use in the area of material science, as reviewed in [1], but is also relevant for the characterization of biological tissues and cells, as reviewed in [2]. The dielectric properties of cells reveal information on size, membrane resistance, membrane capacitance and cytoplasmic conductivity at frequencies below $10 \mathrm{MHz}$ [3]. The presence of subcellular components, such as vacuoles, can also potentially be detected, but at much higher frequencies [4].

Typically, bulk suspensions have been used for characterizing cells [4], and the results represent an average of the population [5]. The population average does not show important details about the heterogeneity of individual cells, details that are needed to develop mathematical descriptions of cellular behavior [6]. Analysis techniques with single-cell resolution have, therefore, become increasingly important to address such questions [7].

The combination of impedance spectroscopy with a microfluidic flow cytometer results in a lab-on-chip device capable of label-free analysis of single cells [8]. Cells or particles are dispersed in a liquid, typically an electrolyte, such as phosphate-buffered saline (PBS), and forced through a microfluidic channel with two pairs of planar electrodes patterned at the top and bottom. An AC voltage is applied to the electrodes causing a current flow. The current change upon passage of a particle is measured differentially and analyzed to determine the dielectric properties.

Gawad et al. showed, how different cell properties influence the impedance spectrum [9]. They found that the cell size contributes to the response at all frequencies, the membrane capacitance at frequencies around $1 \mathrm{MHz}$, and the cytoplasm conductivity at frequencies approaching $10 \mathrm{MHz}$. Cheung et al. used a similar setup, with simultaneous analysis of multiple frequencies to discriminate beads from red blood cells and red-blood-cell ghosts [10]. Schade-Kampmann et al. used an impedance device for not only discriminating between different cell types, but also for analyzing viability and apoptosis of Jurkat cells [11]. Holmes et al. used the method for discriminating the different leukocytes in human blood [12]. They were able to make a differential white blood cell count with results that correlated to those of commercial blood analyzers. Impedance work of other authors has been reviewed by Sun et al. [13].

In this paper, we report on a novel microfluidic impedance cytometer that covers a frequency range from $\mathrm{DC}$ to up to $500 \mathrm{MHz}$. The increased frequency range will allow for characterization of subcellular components, such as vacuoles and the cell nuclei, in addition 


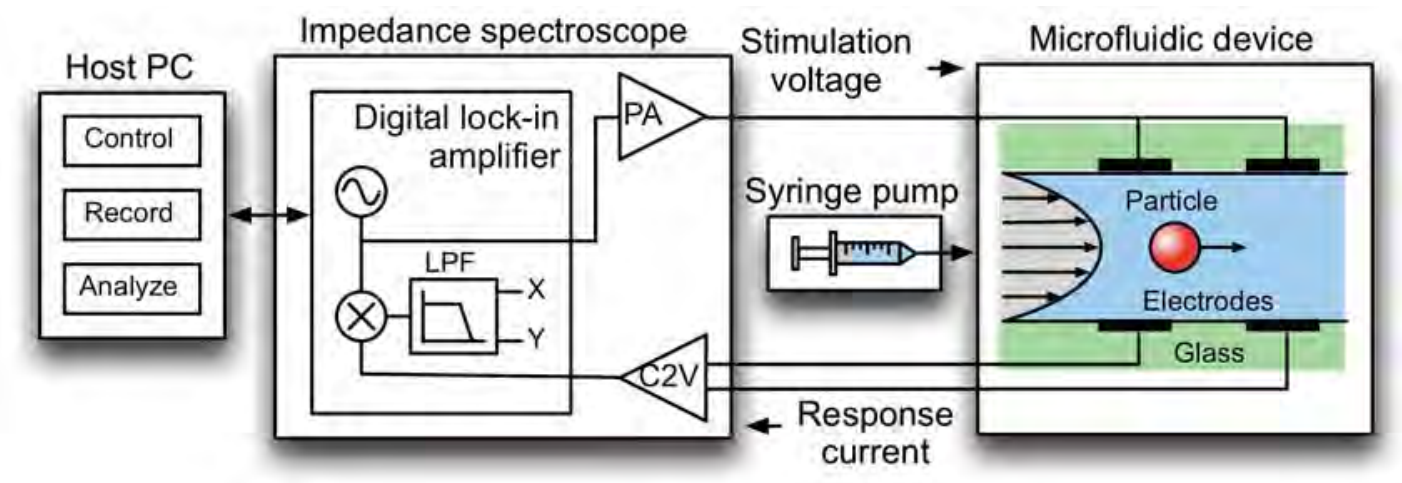

Fig. 1. Schematic diagram showing the complete microfluidic impedance cytometer presented in this paper. A power amplifier (PA) buffers the signal from the impedance spectroscope, and a trans-impedance amplifier $(\mathrm{C} 2 \mathrm{~V})$ converts the response current into a voltage. Cells or particles are dispersed in liquid and forced through the microfluidic channel using a syring pump.

to the properties detectable at lower frequencies. The cytometer consists of a custom-built impedance spectroscope, combined with a simple microfluidic device with facing electrodes. The impedance spectroscope is based on a flexible FPGA platform, which interfaces to the microfluidic device and features high-speed data converters and a trans-impedance amplifier, built from off-theshelf components. We demonstrate the capabilities of the cytometer by discriminating polystyrene beads from Chinese hamster ovary $(\mathrm{CHO})$ cells, based on the difference in dielectric properties at frequencies of up to 500 $\mathrm{MHz}$.

\section{Experimental}

A schematic diagram of the cytometer is shown in Fig. 1. It consists of an impedance spectroscope combined with a microfluidic device. The impedance spectroscope is based on a digital lock-in amplifier (Fig. 2a), which generates the stimulation voltage and analyses the response current. The lock-in amplifier is interfaced to the analog domain by using highspeed data converters operating at $1.8 \mathrm{GSa} / \mathrm{s}$. A power amplifier (Fig. 2b) is used to buffer the signal from the digital-to-analog converter (DAC) before it is applied to the microfluidic device. This is necessary because of the highly frequency-dependent impedance of the microfluidic channel. A trans-impedance amplifier (Fig. 2c) transforms the response current into a voltage, which can be measured by the analog-to-digital converter (ADC). Power and trans-impedance amplifiers are implemented on a dedicated printed circuit board, that also holds the microfluidic device. This keeps the connections between the components short, which minimizes signal reflections and noise coupling into the system.
The digital lock-in amplifier is implemented on a Xilinx Virtex-6 FPGA (Xilinx, Inc., USA). It enables generation and analysis of up to four frequencies in parallel by means of four demodulators.

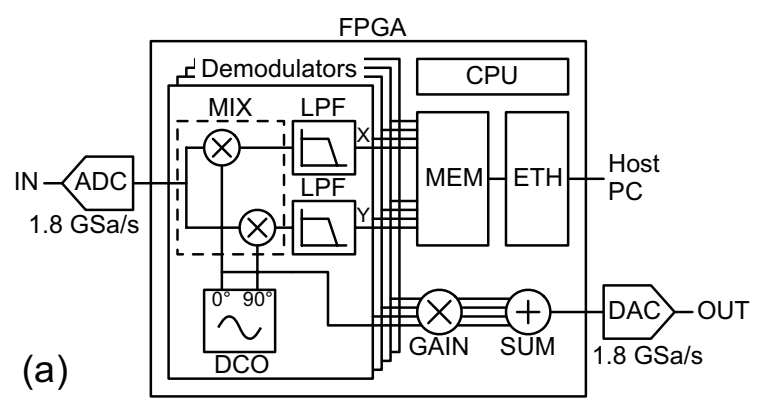

(b)
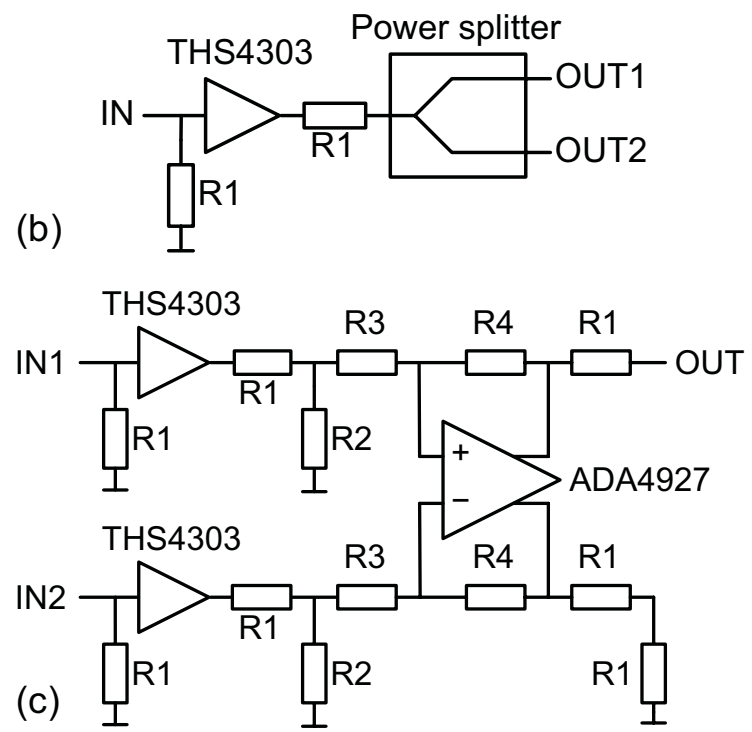

Fig. 2. (a) Block diagram of the digital lock-in amplifier featuring four parallel demodulators. (b) Schematic diagram of the $P A$. (c) Schematic diagram of the C2V. PA and C2V have been implemented using off-the-shelf components. The resistances $R 1$ to $R 4$ are $50 \Omega, 56 \Omega, 301 \Omega$ and $470 \Omega$ respectively. 


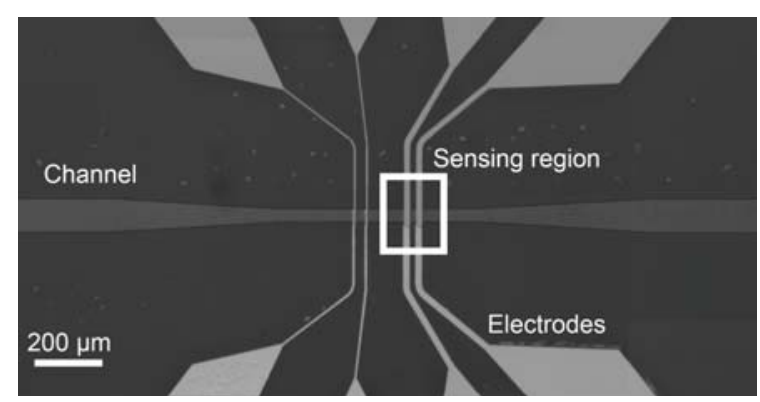

Fig. 3. Micrograph of the microfluidic device. The electrodes are $18 \mu \mathrm{m}$ wide with a center-to-center spacing of $36 \mu \mathrm{m}$. Channel width is $40 \mu \mathrm{m}$ in the sensing region.

Each demodulator has a digitally controlled oscillator (DCO), a frequency mixer (MIX), and two low-pass filters (LPF). The lock-in amplifier output constitutes of a linear combination of the in-phase part of the signals of the four DCOs. Each DCO generates a sinusoidal carrier signal with a frequency, $f_{d c o}$, of up to $500 \mathrm{MHz}$ and a resolution better than $10 \mu \mathrm{Hz}$. The mixer shifts the frequencies of the input signal, $f_{i n}$, to $f_{i n}-f_{d c o}$ and $f_{i n}+f_{d c o}$. The low-pass filters suppress the frequency components at $f_{i n}+f_{d c o}$, but also help to reject noise on the input signal. They feature configurable order, bandwidth and downsampling ratio to enable a flexible trade-off between noise rejection and response time. The samples of the resulting in-phase $(X)$ and $90^{\circ}$ out-of-phase $(\mathrm{Y})$ signals are continuously buffered in the memory, packed in User Datagram Protocol packets and streamed to the host PC over Ethernet. Here, they are stored for off-line analysis. The lock-in amplifier can reliably deliver samples at a total sampling rate of up to $2.8 \mathrm{MSa} / \mathrm{s}$, shared between the four

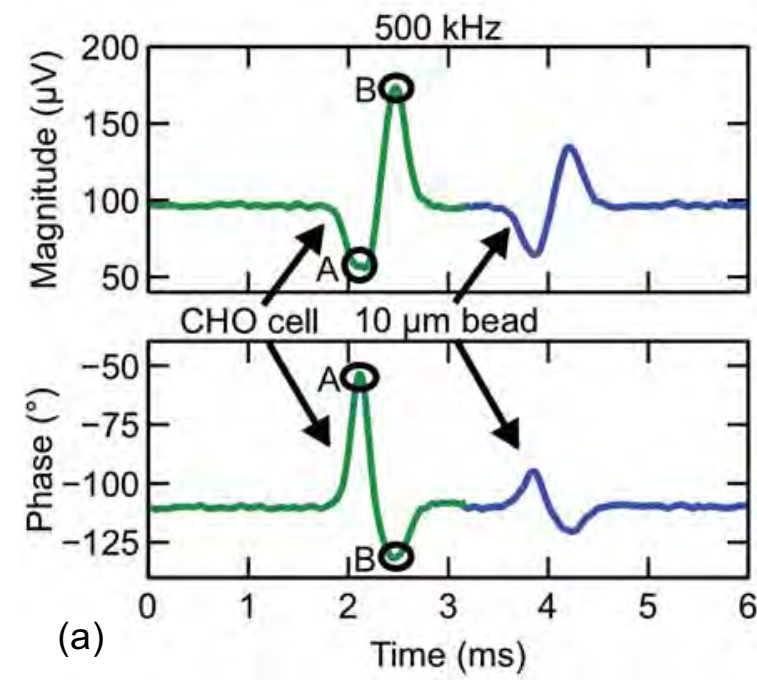

demodulators.

The microfluidic device (Fig. 3) has been fabricated as described in [14]. It consists of two glass plates with $200-n m$-thick and $18-\mu \mathrm{m}$ wide platinum electrodes. The two plates are bonded face to face using a 40- $\mu \mathrm{m}$-thick lithographically structured layer of SU-8 3025 as spacer that defines the channel dimensions. The final channel cross-section in the sensing region is $40 \times 40 \mu \mathrm{m}^{2}$.

For the experiment, CHO-K1 cells (CCL-61, American Type Culture Collection, USA) were cultured using standard conditions in Dulbecco's modified Eagle's medium with the addition of $10 \%$ foetal bovine serum (both from Life Technologies Europe B.V., Switzerland) and $1 \%$ penicillin-streptomycin. The cells were trypsinized and re-suspended in an isotonic sucrose buffer for a final concentration of approximately $100,000 \mathrm{cells} / \mathrm{ml}$. A suspension of $10-\mu \mathrm{m}$ polystyrene beads (Sigma-Aldrich, Switzerland) diluted in PBS (Sigma-Aldrich, Switzerland) to a concentration of approximately 600,000 beads $/ \mathrm{ml}$ was also prepared. The two solutions were mixed and driven through the channel in the microfluidic device using a syringe pump with a flow-rate of $5 \mu \mathrm{l} / \mathrm{min}$. The impedance was analyzed at four frequencies $(500 \mathrm{kHz}, 5 \mathrm{MHz}, 50 \mathrm{MHz}$ and $500 \mathrm{MHz}$ ) by using 3rd order filters with a bandwidth of $10 \mathrm{kHz}$.

\section{Results and discussion}

Fig. 4 shows an example of the recorded impedance signals from the lock-in amplifier for $500 \mathrm{kHz}$ and $500 \mathrm{MHz}$, as a cell and a bead pass the electrodes. The figure shows that a significant impedance change can be detected

Fig. 4. (a) Magnitude and phase of the recorded time-domain impedance signal at $500 \mathrm{kHz}$ showing peaks of a cell and a bead. The peak-to-peak voltage is calculated by complex subtraction of the values at the points marked with $A$ and $B$. (b) Corresponding time-domain signal at $500 \mathrm{MHz}$. 

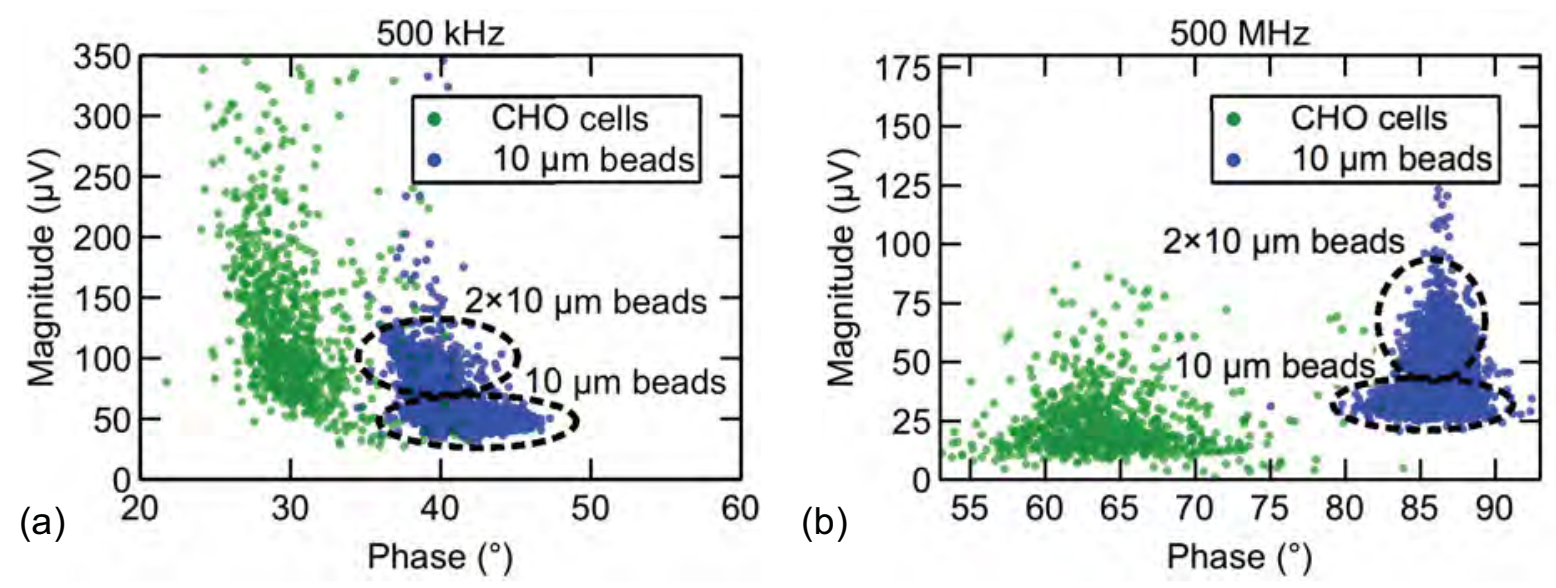

Fig. 5. (a) Impedance results for measurements at $500 \mathrm{kHz}$, where the magnitude is proportional to the object volume. (b) Impedance results at $500 \mathrm{MHz}$ showing distinct responses cells and beads, as a consequence of the difference in their dielectric properties.

for cells at frequencies of up to $500 \mathrm{MHz}$. The impedance signal exhibits a characteristic double-peak, as the cell/bead moves through the sensing region, which is a consequence of the impedance change and the differential measurement principle. The double-peaks were detected using MATLAB (MathWorks, Inc., USA), and a complex peak-to-peak value was extracted for each event by subtracting the values at the points marked "A" and "B". A clustering method was then used to classify each event as either "cell" or "bead", based on the differences in dielectric properties at all four measured frequencies. The resulting complex peak-to-peak values for the complete set of measurements are shown in Fig. 5.

The magnitude of the impedance change at $500 \mathrm{kHz}$ (Fig. 5a) is determined by the volume of the detected object. An overlap between cells and beads can be observed, which shows that they have comparable size. The average cell diameter is calculated to be $12.5 \mu \mathrm{m}$, which is close to reported values for $\mathrm{CHO}$ cells of $12.9 \mu \mathrm{m}$ [15]. A relatively large variation is to be expected, as no effort has been made to control the cell development stage during culturing. Two groups can be observed for the beads. These are caused by single beads, and pairs of beads passing together. The paired beads show the corresponding double impedance signal magnitude compared to single beads.

The response of the cells at $500 \mathrm{MHz}$ (Fig. 5b) is dominated by the dielectric properties of cytoplasm and nucleus, which are comparable to those of the suspension medium. These cellular dielectric characteristics lead to a lower amplitude, and, therefore, a smaller impedance change, compared to that of non-conductive beads.
The phase signal of the impedance change is mainly determined by the capacitance of the detected object. The beads show a larger change at both $500 \mathrm{kHz}$ and $500 \mathrm{MHz}$. This is due to the poor relative permittivity of the polystyrene material, which causes a greater reduction in capacitance of the channel in comparison to the cells.

\section{Conclusion}

We have presented a novel microfluidic impedance cytometer, capable of simultaneous analysis at four frequencies between $\mathrm{DC}$ and $500 \mathrm{MHz}$. This represents a more than ten-fold increase in the frequency range in comparison to other devices.

We have demonstrated that relevant impedance characteristics can be measured for single cells as well as for beads over the full frequency range of the cytometer. The results at $500 \mathrm{kHz}$ revealed the average cell diameter to be $12.5 \mu \mathrm{m}$, which is close to what has been reported by other authors. The results at $500 \mathrm{MHz}$ showed the responses of cells and beads to be very distinct, due to the difference in dielectric properties. The cells gave rise to a smaller response in comparison to the beads. This was attributed to the higher conductivity of the cell cytoplasm and nucleus, which dominates the response at high frequencies.

Current work is ongoing to use the high frequency capabilities of the cytometer to differentiate cells according to differences in internal morphology.

\section{Acknowledgements}

The research leading to these results has received funding from the Commission for Technology and Innovation CTI, Switzerland under project no. 11174.2 PFLS-LS. 


\section{References}

[1] I. D. Raistrick, Application of Impedance Spectroscopy to Materials Science, Annual Review of Materials Science 16, 343-370 (1986); doi: 10.1146/annurev.ms.16.080186.002015

[2] K. R. Foster and H. P. Schwan, Dielectric properties of tissues and biological materials: a critical review., Critical reviews in biomedical engineering 17, 25-104 (1989); PMID: 2651001

[3] R. Pethig and D. B. Kell, The passive electrical properties of biological systems: their significance in physiology, biophysics and biotechnology, Physics in Medicine and Biology 32, 933-970, (1987); doi: 10.1088/0031-9155/32/8/001

[4] K. Asami, T. Yonezawa, Dielectric behavior of wild-type yeast and vacuole-deficient mutant over a frequency range of $10 \mathrm{kHz}$ to $10 \mathrm{GHz}$., Biophysical journal 71, 2192-200 (1996); doi: 10.1016/S0006-3495(96)79420-1

[5] D. Di Carlo and L. P. Lee, Dynamic Single-Cell Analysis for Quantitative Biology, Analytical Chemistry 78, 7918-7925 (2006); doi: 10.1021/ac069490p

[6] M. E. Lidstrom and D. R. Meldrum, Life-on-achip., Nature reviews. Microbiology 1, 158-64, (2003); doi 10.1038/nrmicro755

[7] H. A. Svahn and A. van den Berg, Single cells or large populations?, Lab on a chip 7, 544-6, (2007); doi: 10.1039/b704632b

[8] S. Gawad, L. Schild, and P. H. Renaud, Micromachined impedance spectroscopy flow cytometer for cell analysis and particle sizing., Lab on a chip 1, 76-82, (2001); doi: 10.1039/b103933b

[9] S. Gawad, K. Cheung, U. Seger, A. Bertsch, and $P$. Renaud, Dielectric spectroscopy in a micromachined flow cytometer: theoretical and practical considerations., Lab on a chip 4, 241-51 (2004); doi 10.1039/b313761a

[10] K. Cheung, S. Gawad, and P. Renaud, Impedance spectroscopy flow cytometry: on-chip label-free cell differentiation, Cytometry. Part A : the journal of the International Society for Analytical Cytology 65, 124-32 (2005); doi 10.1002/cyto.a.20141

[11] G. Schade-Kampmann, a Huwiler, M. Hebeisen, T. Hessler, and M. Di Berardino, On-chip noninvasive and label-free cell discrimination by impedance spectroscopy., Cell proliferation 41, 830-40 (2008); doi: 10.1111/j.13652184.2008.00548.x

[12] D. Holmes, D. Pettigrew, C. H. Reccius, J. D. Gwyer, C. van Berkel, J. Holloway, D. E. Davies, and $\mathrm{H}$. Morgan, Leukocyte analysis and differentiation using high speed microfluidic single cell impedance cytometry., Lab on a chip 9, 2881-2889 (2009); doi 10.1039/b910053a

[13] T. Sun, H. Morgan, Single-cell microfluidic impedance cytometry: a review, Microfluidics and
Nanofluidics 8, 423-443 (2010);

doi: $10.1007 / \mathrm{s} 10404-010-0580-9$

[14] S. C. Bürgel, Z. Zhu, N. Haandbaek, O. Frey, and A. Hierlemann, Dynamic and static impedance spectroscopy for single particle characterization in microfluidic chips, in 2012 IEEE 25th International Conference on Micro Electro Mechanical Systems (MEMS), 1033-1036 (2012); doi: 10.1109/MEMSYS.2012.6170247

[15] W. Pilbrough, T. P. Munro, and P. Gray, Intraclonal protein expression heterogeneity in recombinant $\mathrm{CHO}$ cells., PloS one 4, e8432 (2009); doi:10.1371/journal.pone.0008 